\title{
ADAPTACIÓN AL ESPAÑOL DE LA ESCALA DE ACTITUDES ANTE CUIDADOS PALIATIVOS: CONFIABILIDAD Y ANÁLISIS FACTORIAL
}

\author{
SPANISH ADAPTATION OF THE SCALE OF ATTITUDES TOWARDS PALLIATIVE CARE: \\ RELIABILITY AND FACTOR ANALYSIS
}

Leticia Ascencio Huertas

Servicio de Cuidados Paliativos del Instituto Nacional de Cancerología, b. Doctorado en Ciencias Sociales y administrativas de la Universidad Chapultepec

Resumen

Este estudio instrumental presenta los criterios de confiabilidad y análisis factorial como parte de la adaptación al español de la Escala de Actitudes ante los Cuidados Paliativos (EACP). Se realizó con el objetivo de conocer si la escala cuenta con las propiedades psicométricas para poder ser utilizado para evaluar las actitudes que tiene el personal de salud hacia los cuidados paliativos. La muestra estuvo compuesta por 132 profesionales de la salud (80,3\% mujeres), con edades comprendidas entre los 20 y 40 años $(M=$ 30; DT = 9,11). Los análisis exploratorios y confirmatorios mostraron un índice de fiabilidad satisfactorio (Alfa de Cronbach: 0,807 ) que expliquen el $65,063 \%$ de la varianza y la solución factorial que contiene las correlaciones entre las variables originales y cada uno de los factores. Dentro de los factores más relevantes consideramos: El Factor 1 que hacen referencia al proceso o directrices de atención en las unidades de Cuidados Paliativos, el Factor 2 que se relacionan con las experiencias personales ante la muerte; $y$ el Factor 5 que se refieren al apoyo social que tienen los Cuidados Paliativos. Concluyendo que la escala cumple con las propiedades psicométricas para ser utilizada tanto en el campo clínico como en la investigación.

\section{Abstract}

This instrumental study presents the reliability and factor analysis as part of the Spanish adaptation of the Scale of Attitudes Palliative Care (PSST). I was done with the aim of knowing if the scale has psychometric properties, to be used to assess attitudes with health staff towards palliative care. The sample was of 132 health professionals (80.3\% women), aged between 20 and 40 years $(M=30, S D=9.11)$. Exploratory and confirmatory analysis showed a satisfactory rate of reliability (Cronbach's alpha: 0.807 ) to explain the $65,063 \%$ of the variance and factorial solution containing the correlations between the original variables and each of the factors. Among the most important factors we consider: Factor 1 refers to the process and guidelines for care units Palliative Care Factor 2 that relate personal experiences with death; and Factor 5 which relate to social support have Palliative Care. Concluding that the scale fulfills the psychometric properties for use in both clinical and research field.

Keywords: Attitudes, palliative care.

Palabras Clave: Actitudes, cuidados paliativos.

Correspondencia:

Leticia Ascencio Huertas.

Servicio de Cuidados Paliativos del Instituto Nacional de Cancerología.

Av. San Fernando No. 22, Col. Sección XVI. Delegación Tlalpan, C.P. 14080 México,

E-mail: leash71@yahoo.com.mx 


\section{INTRODUCCIÓN}

Los especialistas en el campo de la salud, se ven envueltos en diversos y rápidos cambios debidos al auge de la medicina institucional y a las innovaciones tecnológicas, lo que tiende a despersonalizar la práctica médica, haciendo del paciente un simple expediente clínico. Estos cambios no sólo inciden en la práctica médi$\mathrm{ca}$, sino en las interacciones y el contexto en que se dan.

Los pacientes, sus familiares y los mismos profesionales de la salud parecen sentirse cada vez más molestos frente a la medicina que se centra en la enfermedad y se olvida del hombre enfermo. El enfermo en ocasiones se ve envuelto en diversas técnicas diagnósticas, tratamientos cada vez más complejos e instituciones en las que se orienta con dificultad, y que demanda otro tipo de comunicación y de cuidados, pero cada vez el propio paciente solicita información acerca de las alternativas que se presentan frente a la enfermedad, desea elegir entre las prácticas a las que se ha de ser sometido y reflexiona acerca de los limites terapéuticos y el proceso de muerte, lo cual promueve una medicina más participativa entre paciente y médico.

Sabater $^{(1)}$ considera que una buena educación para los cuidados paliativos y la muerte deberían de formar parte de la educación en el personal de salud, ya que desde su punto de vista, deberíamos aceptar a la muerte como un acontecimiento seguro de nuestra existencia.

Por lo que es importante poder transmitir a la sociedad que la Medicina seguirá impidiendo la muerte, pero que además deben poner todos sus esfuerzos en mantener la calidad de vida de sus pacientes. Como seres humanos, además de los médicos, todos tenemos la obligación de exigirnos mutuamente que nuestros semejantes no mueran en la desesperación, la soledad o la indignidad (2).
Uno de los fenómenos a los que los profesionales de la salud se enfrentan en el día a día, es al Ilamado "máximo beneficio", situación cada vez más frecuente por el incremento en las enfermedades crónico-degenerativas y sus complicaciones, que se desarrollan como enfermedades terminales. Así, Ilega la necesidad de afrontar, junto con nuestros pacientes, la inminencia de la muerte, en las mejores condiciones clínicas, psicológicas, sociales y espirituales posibles, por lo que se crean los cuidados paliativos.

La $\mathrm{OMS}^{(3)}$ define Cuidados Paliativos, como el enfoque que mejora la calidad de vida de pacientes y familias que se enfrentan a los problemas asociados con enfermedades amenazantes para la vida, a través de la prevención y el alivio del sufrimiento por medio de la identificación temprana y la impecable evaluación y tratamiento del dolor y otros problemas físicos, psicosociales y espirituales. Es importante resaltar que un paciente susceptible de recibir cuidados paliativos es aquel que cursa con una enfermedad o condición avanzada crónica e incurable que puede incluir trastornos infecciosos, traumáticos, metabólicos, degenerativos y oncológicos independientemente del pronóstico de tiempo de sobrevida ${ }^{(4)}$.

En congruencia con las necesidades de la población mexicana y la búsqueda de mejores opciones para los pacientes en estado terminal, debe adoptarse un nuevo enfoque integral en nuestro sistema nacional de salud. Éste debe disminuir las penurias físicas, emocionales y espirituales que enfrentan los enfermos ${ }^{(5,6)}$.

La atención a los pacientes más graves y terminales requiere el conocimiento y la formación de médicos y otros profesionales de la salud, que actúen acorde a los objetivos de esta nueva área: proporcionar una atención integral y holística, lo que nos lleva a considera que uno de los puntos relevantes en esta profesionaliza- 
ción es conocer las actitudes que tienen el propio personal de salud ante los cuidados paliativos.

Estudios como los realizados por Weber, Schmiedel, Nauck \& Alt-Epping( ${ }^{(7)}$, Bowden, Dempsey, Boyd, Fallon \& Murray ${ }^{(8)}$, Snyder, Hazelett, Allen \& Radwany ${ }^{(9)}$, Chen, McCann \& Lateef ${ }^{(10)}$, Kavalieratos, et al. ${ }^{(11)}$, Schaefer, et al. ${ }^{(12)}$ sugieren que los cuidados paliativos son importantes y se requieren de mayor preparación para los médicos de pregrado y de posgrado para desarrollar habilidades, conocimientos y mejorar la actitud que tienen de los cuidados paliativos y la atención al final de la vida así como de la importancia de la comunicación y del manejo del dolor y cambiar la actitud que tienen los médicos especialistas de los cuidados paliativos (Twamley, et al. ${ }^{(13)}$; Dietz, et al.(14); Loucka, Pasman, Brearley, Payne, \& Onwuteaka-Philipsen ${ }^{(15)}$ logrando así mejorar los recursos suficientes y necesarios para brindar cuidados paliativos a los pacientes que por su condición de salud así lo requieran y su calidad de vida ${ }^{(16)}$.

Existen algunos estudios que describen el actitudes hacia la atención del paciente con cáncer al final de la vida, como el realizado por Cherny y Catane ${ }^{(17)}$ que analizaron las actitudes de los oncólogos al paciente oncológico terminal, encontrando que más del $75 \%$ de los oncólogos acordaron que todos los pacientes con cáncer avanzado deben recibir cuidados paliativos y que todos los centros de atención a pacientes con cáncer deben tener un servicio de cuidados paliativos ( $89,1 \%)$; que los oncólogos médicos deben coordinar el cuidado de los pacientes con cáncer en todas las etapas de la enfermedad, incluida la atención al final de su vida $(88,4 \%)$; y que debe ser experto en la gestión de la síntomas físicos y psicológicos de avanzada cáncer (88,1\%); con- cluyendo que las actitudes son dignas de atención especial en la formación de los médicos oncólogos, ya que no están incluidos los cuidados paliativos durante su entrenamiento profesional, y que la mayoría de los oncólogos tienen una baja preparación para controlar los síntomas físicos y psicológicos del cáncer avanzado. Por otro lado, Cortezzo, Sanders, Brownell y Moss ${ }^{(18)}$ analizaron la perspectiva y actitudes que tienen los neonatolgos con respecto a los cuidados paliativos y los cuidados intensivos neonatales, reportando que las barreras para poder implementar los servicios de cuidados paliativos eran las dificultades emocionales, los desacuerdos y dificultades en la formación de equipos de cuidados paliativos.

Un punto relevante con respecto a las actitudes sobre los cuidados paliativos es que no existen instrumentos de evaluación que cumplan con los criterios psicométricos necesarios. Al respecto, Frey, Gott, Banfield y Campbell ${ }^{(19)}$ realizaron una revisión sistemática de la literatura cualitativa y cuantitativa durante el período 1990-2010, con el objetivo de determinar si existe un cuestionario para medir la percepción de competencias generales en la prestación de los cuidados paliativos en Medline, Medline in Progress, bases de datos PubMed y CINAHL, así como en manuales de Medicina Paliativa, la Revista Internacional de Enfermería Paliativos y el Diario de Cuidados Paliativos, concluyendo que, no hay un solo cuestionario validado para medir la percepción de competencia en la gestión de los cuidados paliativos entre los profesionales de la salud, y que en general, solo existe un foco sobre los aspectos físicos del tratamiento de los síntomas en cuidados paliativos.

Sin embargo, al realizar una búsqueda exhaustiva en la literatura especializada, se encontraron solo algunos instrumentos 
relacionados con las actitudes y creencias acerca de los cuidados paliativos. Uno de ellos es Attitudes to Palliative Care Practice in Neonatal Intensive Scale (NiPCAS) desarrollado por Kain ${ }^{(20)}$, quien diseña este instrumento para medir las actitudes hacia la práctica de cuidados paliativos en el personal de enfermería de cuidados intensivos neonatales y que fue reportado por Kain, Gardner y Yates ${ }^{(21)}$ y que posteriormente fue adaptada por Kumar, Jim, \& Sisodia( ${ }^{(22)}$ con estudiantes de fisioterapia, adquiriendo el nombre de PTiPCKABE Scale y utilizado por Chen, Huang, Liu., Wu, Chang y Peng ${ }^{(23)}$ para analizar las actitudes y creencias con enfermeras neonatales.

Otro instrumento es el construido por Al-Kindi, Zeinah y Hassan ${ }^{(24)}$, que evalúa conocimientos y habilidades en Cuidados Paliativos; consta de 29 preguntas divididas en 4 secciones: formación previa y conocimientos en cuidados paliativos, evaluación de auto-competencia en el tratamiento de los pacientes que mueren con cáncer, manejo de los síntomas y las actitudes hacia el cuidado al final de la vida. Sin embrago solo ha sido aplicada en una población específica y no se reportan las propiedades psicométricas del cuestionario. Y la escala Frommelt Attitude Toward Care of the Dying (FATCOD) desarrollada por Frommelt ${ }^{(25,26)}$ y que detecta específicamente en estudiantes de enfermería actitudes para el cuidado de los pacientes que mueren ${ }^{(27)}$, sin embargo, no se encontraron estudios que reporten la validez y fiabilidad de esta escala.

Razón por la que decidimos traducir y analizar la confiabilidad y el análisis factorial de la Escala de Actitudes ante los Cuidados Paliativos; que de acuerdo a la literatura ha sido utilizados en 3 poblaciones: enfermeras de cuidados intensivos neonatales Kain ${ }^{(20,21)}$, estudiantes de fisioterapia $^{(22)}$ y enfermeras neonatales ${ }^{(23)}$.

\section{MÉTODO}

\section{Diseño}

No experimental, retrospectivo y transversal.

\section{Muestreo}

No probabilístico, de tipo intencional o de conveniencia

\section{Participantes}

En este estudio participaron 150 profesionales de la salud (médicos, psicólogos, enfermeras, trabajadores sociales, odontólogos) de diversas instituciones públicas de salud de la República Mexicana, de los cuales 18 fueron eliminados por que los cuestionarios estaban incompletos, no firmaron consentimiento informado o dejaron en blanco los cuestionarios, por lo que la muestra total fue de 132 participantes.

\section{Instrumentos de evaluación}

La Escala de Actitudes ante Cuidados Paliativos (EACP) La EACP fue creada por Kain ${ }^{(20)}$ para medir las actitudes hacia la práctica de cuidados paliativos en las unidades de cuidados intensivos neonatales en el personal de enfermería: The Neonatal Palliative Care Attitude Scale (NiPCAS) que consta de 33 reactivos, de los cuales 7 son para identificar características sociodemográficas y profesionales y 26 de escala tipo Likert de 5 rubros (Totalmente de acuerdo, de acuerdo, neutro, en desacuerdo, totalmente en desacuerdo) con un alfa de Cronbach 0,80(20,21); y modificado por Kumar, Anand y Sisodia (2011) (22) para valorar los conocimientos, actitudes, creencias y experiencias en cuidados paliativos en fisioterapeutas (The physical therapy in palliative care-knowledge, at- 
titudes, beliefs and experiences scale, PTiPC-KABE Scale), que consta de 37 reactivos totales: 7 para identificar características sociodemográficas y profesionales, 26 con escala likert de 5 rubros: 1 (totalmente en desacuerdo), 2 (desacuerdo), 3 (neutro, no estoy seguro), 4 (de acuerdo) y 5 (totalmente de acuerdo). Y cuatro reactivos de preguntas abiertas; obteniéndose un alfa de Cronbach total en esta versión de 0,90.

La versión final que se adaptó, consta de un apartado inicial para recabar la información relacionada con las características sociodemográficas y profesionales (nombre, edad, género, estado civil, ocupación, grado de estudios, años de experiencia clínica, puesto) que a diferencia de la versión original no se consideraron como reactivos sino como ficha de identificación, y la sección 2 de la prueba original de Kain ${ }^{(20,21)}$ que son 26 reactivos con tipo de respuesta tipo Likert de 5 rubros (Totalmente de acuerdo, de acuerdo, neutro (no estoy seguro), en desacuerdo, totalmente en desacuerdo) y dos reactivos de tipo cualitativo (preguntas abiertas): el primer reactivo describe el interés general que tiene el profesional de la salud hacia los cuidados paliativos, y el segundo reactivo nos permite identificar el significado de los Cuidados Paliativos para cada uno de los participantes.

\section{Procedimiento}

Para analizar la confiabilidad y análisis factorial de este instrumento en la versión en español, se llevó a cabo la adaptación y evaluación de la confiabilidad de la siguiente manera: Se procedió inicialmente con la traducción del instrumento Neonatal Palliative Care Attitude Scale propuesta por Kain ${ }^{(20,21)}$, ya que la versión original se encuentra en el idioma inglés y que basó en el método de traducción directa y la traducción inversa ${ }^{(28)}$, a través de
5 jueces bilingües (3 español-inglés y 2 inglés-español). Posteriormente a 5 jueces expertos en el área de la salud específicamente de Cuidados Paliativos, quienes verificaron el estilo, redacción, originalidad y estructuración del instrumento en español. Una vez realizada la traducción se efectúo la prueba piloto con 25 aplicaciones para verificar la validez de constructo y de contenido, de acuerdo a los lineamientos de Aiken ${ }^{(29)}$.

Cada uno de los participantes aceptó participar en el presente estudio mediante la comprensión y firma del consentimiento informado.

\section{RESULTADOS}

Se describieron las características sociodemográficas de la muestra mediante medidas de tendencia central, de dispersión y tablas de frecuencia y porcentaje. Se determinó la estructura factorial por medio de un análisis factorial con rotación varimax y método de extracción a través del paquete estadístico SPSS versión 19.

Con respecto a las características sociodemográficas los resultados muestran que la población estudiada estuvo conformada por 132 participantes de los cuales el $80,3 \%$ fueron mujeres y el 19,7 hombres; el $70,5 \%$ fueron solteros, el $25.9 \%$ casados, el 3,0\% vivía en pareja (unión libre) y el 0,8 \% eran divorciados; con respecto al grado de estudios fueron en su mayoría de nivel licenciatura con el $73,5 \%, 10,6 \%$ con grado de Maestría, el 7,6 con grado de Especialidad, 6,1\% con Subespecialidad, 1,5\% a nivel técnico y $0,8 \%$ con Doctorado. El 46,2\% fue personal de base en alguna institución de salud, el 24,2\% realizaban servicio social, el 12,1\% fueron médicos residentes, el $9,8 \%$ estudiantes, 3,8\% directivos, 3\% fueron trabajadores eventuales y $0,8 \%$ profesionales independientes. Con res- 
pecto al tiempo de experiencia clínica se consideraron aquellos profesionales de salud que tuvieran seis meses como mínimo realizando actividades asistenciales. La tabla 3, nos muestra como el 65,2\% contó con una experiencia de 6 meses a 5 años, el $15,2 \%$ de 6 a 10 años, y el 10,6\% de 11 a 15 años (Ver tabla 1).

Con una edad promedio de 30,03 años, mediana de 27,50, moda de 3 , desviación típica de 9,111, varianza de 83,007, con un mínimo de edad de 20 y máximo de 60 años. Siendo más frecuente el rango de edad de 20 a 25 años con $41,7 \%$ y de 26 a 30 años con el 20,5\% como puede verse en la tabla 2 .

En la tabla 3 se muestran los estadísticos de resumen de los elementos, tanto las medias de los elementos, como las varianzas de los elementos.

\section{Tabla 1. Características de la muestra}

\section{Características sociodemográficas}

\section{F}

\section{Sexo}

Femenino

Masculino

\section{Estado Civil}

Solter@

Casad@

Divorciad@

Unión Libre

\section{Disciplina}

Psicología

Medicina

Enfermería

Trabajo Social

Odontología

\section{Grado de Estudios}

Licenciatura

Maestría

Doctorado

Especialidad

Subespecialidad

Técnico

106

26

\section{3}

34

1

4
80,3

19,7

70,5

25,8

0,8

3,0

\section{Años de Experiencia Clínica}

6 meses a 5 años

6 a 10 años

11 a 15 años

16 a 20 años

21 a 25 años 
Tabla 2. Frecuencia y porcentajes de rangos de edad y Medidas de tendencia central y de dispersión

\begin{tabular}{ccclr}
\hline Rangos de edad & $\mathbf{F}$ & $\mathbf{\%}$ & \multicolumn{2}{c}{ Medidas de tendencia central } \\
\hline 20 a 25 años & 55 & 41,7 & Media & 30,03 \\
26 a 30 años & 27 & 20,5 & Mediana & 27,50 \\
31 a 35 años & 20 & 15,2 & Moda & 23 \\
36 a 40 años & 11 & 8,3 & Desv. típ. & 9,111 \\
41 a 45 años & 11 & 8,3 & Varianza & 83,007 \\
46 a 50 años & 2 & 1,5 & Rango & 40 \\
51 a 55 años & 2 & 1,5 & Mínimo & 20 \\
56 a 60 años & 4 & 3,0 & Máximo & 60 \\
$\quad$ N= & 132 & 100,0 & & \\
\hline
\end{tabular}

Tabla 3. Estadísticos de resumen de los elementos

\begin{tabular}{|lcc|c|c|c|c|c|}
\cline { 2 - 7 } \multicolumn{1}{c|}{} & Media & Mínimo & Máximo & Rango & $\begin{array}{c}\text { Máximo/ } \\
\text { mínimo }\end{array}$ & Varianza & $\begin{array}{c}\text { N de } \\
\text { elementos }\end{array}$ \\
\hline $\begin{array}{l}\text { Medias de los } \\
\text { elementos }\end{array}$ & 3,132 & 1,871 & 4,553 & 2,682 & 2,433 &, 452 & 26 \\
$\begin{array}{l}\text { Varianzas de } \\
\text { los elementos }\end{array}$ & 1,535 &, 936 & 4,123 & 3,187 & 4,404 &, 354 & 26 \\
\hline
\end{tabular}

\section{Confiablidad}

Para calcular el Alfa de Cronbach se consideraron solo los reactivos cuantitativos (del reactivo 2 al 27) ya que los reactivos 1 y 28 son de tipo cualitativo; obteniendo un Alfa total de 0,807 , lo cual comprueba la validez de la Escala, obteniendo una media de 81,43, varianza de 178,003, deviación típica de 13,342 de los 26 reactivos evaluados.

En la tabla 4 se muestra el coeficiente de homogeneidad corregido en el cual se puede observar que si se eliminará el reactivo 18, el Alfa de Cronbach aumentaría a 0,828 , sin embargo consideramos que el Alfa de Cronbach obtenido con los 26 reactivos es aceptable para confirmar la confiabilidad de la Escala al ser de 0,807. Cabe mencionar que en la versión en español se retomó la segunda parte de la escala original (Neonatal Palliative Care Attitude Scale) ${ }^{(20)}$ que cuentan con 26 reac- tivos tipo likert y se agregaron 2 reactivos más de tipo cualitativo (reactivo 1 y 28).

\section{Análisis factorial}

La factorabilidad de los datos se confirmó con las correlaciones mayores a 0.30; la prueba de esfericidad de Bartlett $=1222,554, \mathrm{gl}=325$, nivel de significancia de 0,00 y el Kaiser-Meyer-Oklin (KMO) que fue de 0,807 , por lo que el análisis factorial está confirmado.

La tabla 5 muestra como la suma de cuadrados de la columna total nos ayudó a determinar los 6 de factores que se extrajeron para poder explicar el $65,063 \%$ de la varianza total.

En la tabla 6 se encuentra la solución factorial que contiene las correlaciones entre las variables originales (o saturaciones) y cada uno de los factores. Dentro de los factores más relevantes consideramos únicamente aquellos que tienen 2 o más 


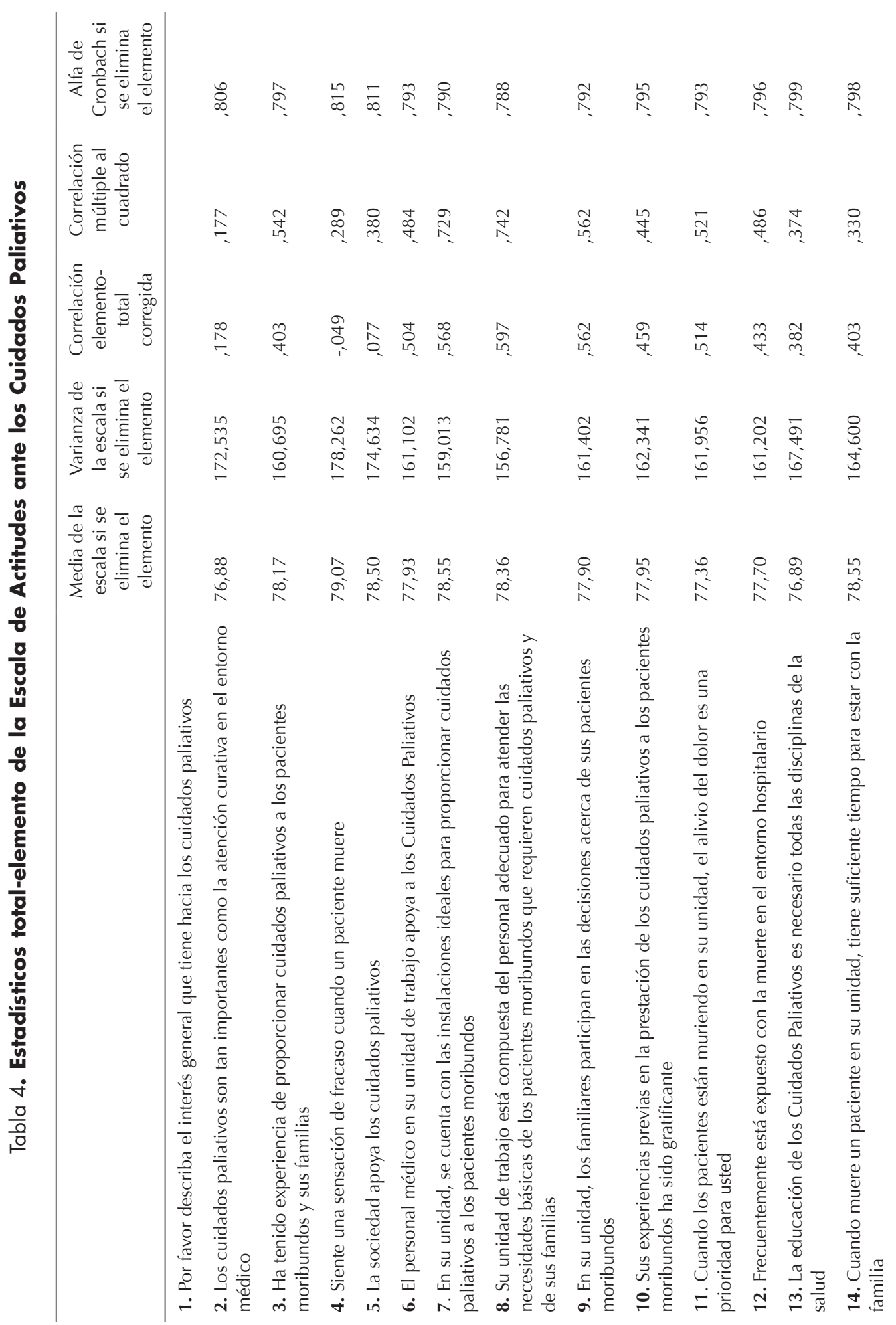




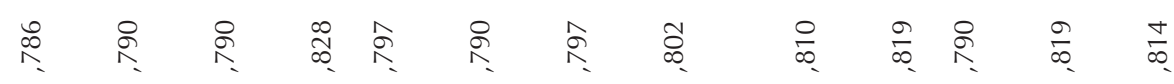

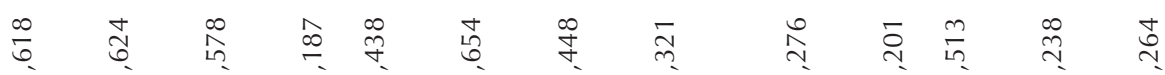

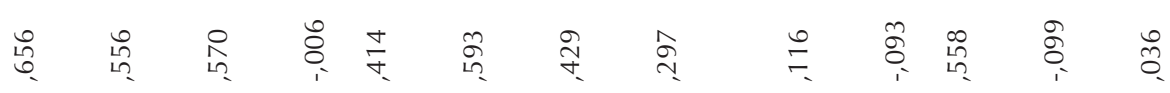

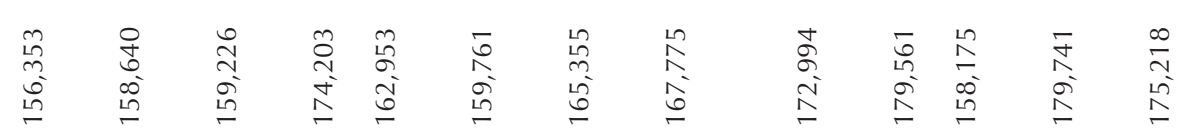

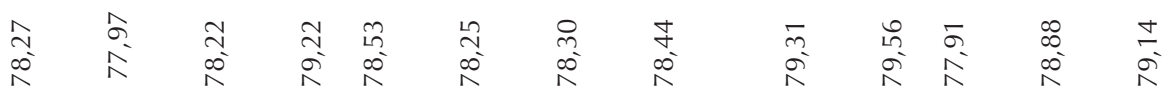

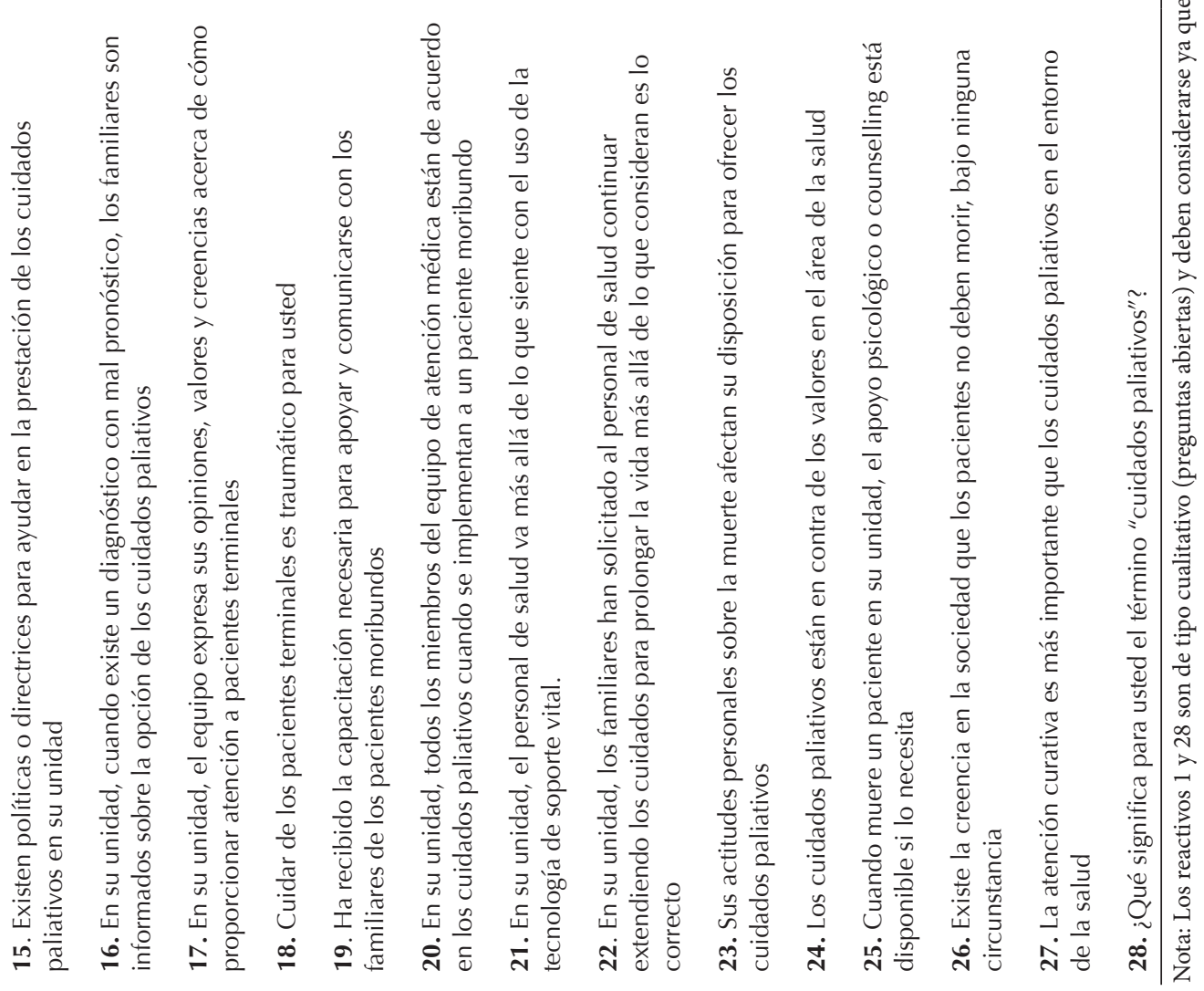




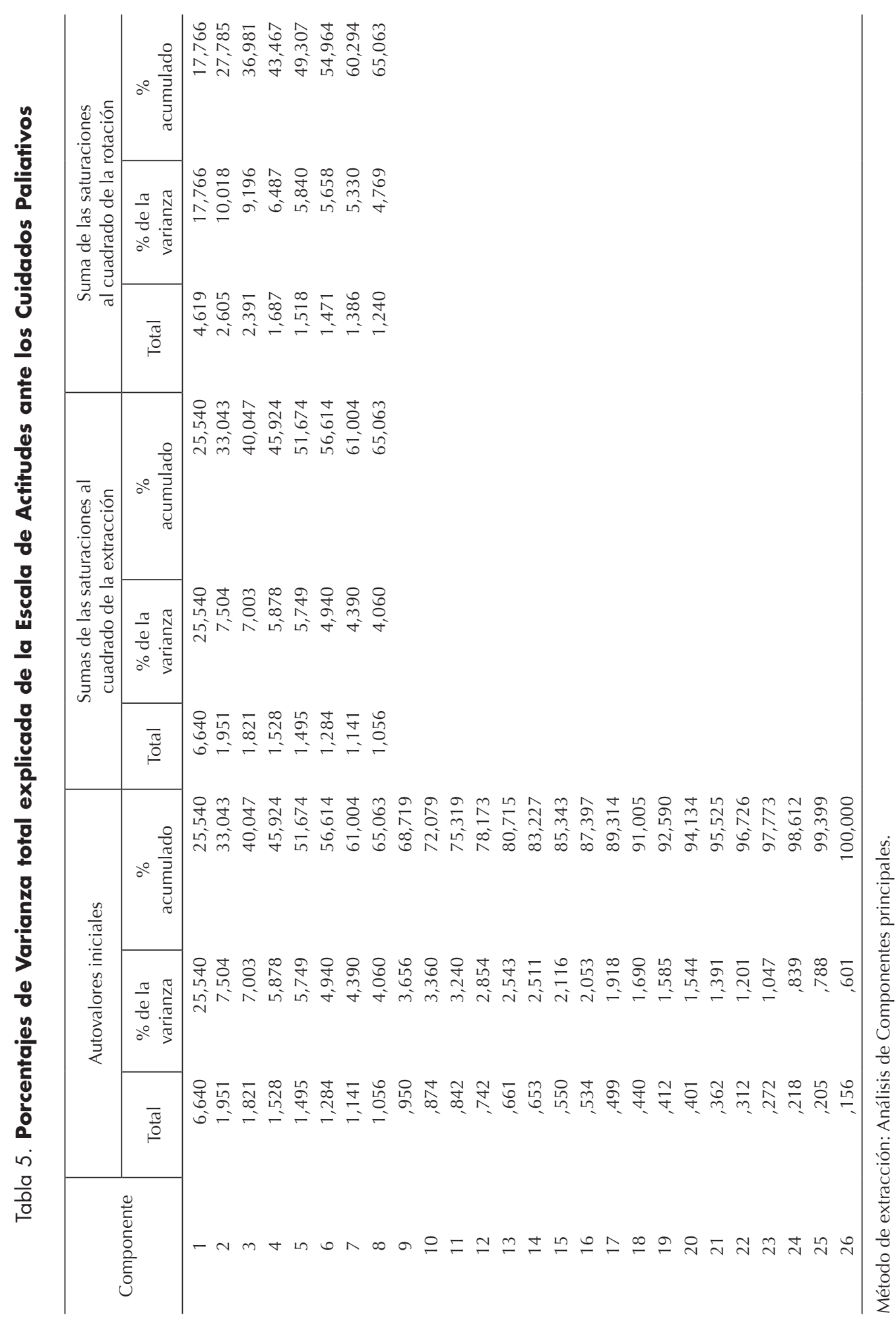




\section{Tabla 6. Análisis de Factor de la Escala de Actitudes ante los Cuidados Paliativos}

\section{Reactivos}

8. Su unidad de trabajo está compuesta del personal adecuado para atender las necesidades básicas de los pacientes moribundos que requieren cuidados paliativos y de sus familias

9. En su unidad, los familiares participan en las decisiones acerca de sus pacientes moribundos

7. En su unidad, se cuenta con las instalaciones ideales para proporcionar cuidados paliativos a los pacientes moribundos

20. En su unidad, todos los miembros del equipo de atención médica están de acuerdo en los cuidados paliativos cuando se implementan a un paciente moribundo

16. En su unidad, cuando existe un diagnóstico con mal pronóstico, los familiares son informados sobre la opción de los cuidados paliativos

17. En su unidad, el equipo expresa sus opiniones, valores y creencias acerca de cómo proporcionar atención a pacientes terminales

15. Existen políticas o directrices para ayudar en la prestación de los cuidados paliativos en su unidad

25. Cuando muere un paciente en su unidad, el apoyo psicológico o counselling está disponible si lo necesita

6. El personal médico en su unidad de trabajo apoya a los Cuidados Paliativos

14. Cuando muere un paciente en su unidad, tiene suficiente tiempo para estar con la familia

3. Ha tenido experiencia de proporcionar cuidados paliativos a los pacientes moribundos y sus familias

12. Frecuentemente está expuesto con la muerte en el entorno hospitalario

10. Sus experiencias previas en la prestación de los cuidados paliativos a los pacientes moribundos ha sido gratificante

11. Cuando los pacientes están muriendo en su unidad, el alivio del dolor es una prioridad para usted

13. La educación de los Cuidados Paliativos es necesario todas las disciplinas de la salud

21. En su unidad, el personal de salud va más allá de lo que siente con el uso de la tecnología de soporte vital.

22. En su unidad, los familiares han solicitado al personal de salud continuar extendiendo los cuidados para prolongar la vida más allá de lo que consideran es lo correcto

26. Existe la creencia en la sociedad que los pacientes no deben morir, bajo ninguna circunstancia

5. La sociedad apoya los cuidados paliativos

27. La atención curativa es más importante que los cuidados paliativos en el entorno de la salud

24. Los cuidados paliativos están en contra de los valores en el área de la salud

23. Sus actitudes personales sobre la muerte afectan su disposición para ofrecer los cuidados paliativos

18. Cuidar de los pacientes terminales es traumático para usted

4. Siente una sensación de fracaso cuando un paciente muere
Factores

$\begin{array}{llllll}1 & 2 & 3 & 4 & 5 & 6\end{array}$

, 567

, 864

586

,308

,304

, 730

, 678

, 548

, 446

,605

, 577 
reactivos, obteniendo un total de 6 Factores: El Factor 1 con 10 reactivos y que hace referencia al proceso o directrices de atención en las unidades de Cuidados Paliativos, el Factor 2 con 5 reactivos que se relaciona con las experiencias personales ante la muerte, el Factor 3 con 3 reactivos que se relaciona con involucrar a la familia en la toma de decisiones y al momento de la muerte del paciente, el Factor 4 con 3 reactivos y que tiene relación con los aspectos sociales frente a los cuidados paliativos, el Factor 5 con 3 reactivos que se refiere a los valores y actitudes ante los Cuidados Paliativos y el factor 6 con 2 reactivos que considera a la muerte como un fracaso o un trauma en el personal de salud.

En la figura se muestra la agrupación de los reactivos de la Escala en espacio rotado en 3 dimensiones, por lo que la calidad de representación de una variable será tanto más alta cuanto mayor sea su distancia al origen del sub espacio generado por las tres dimensiones.

\section{CONCLUSIONES Y DISCUSIÓN}

Cabe señalar que más del $80 \%$ de la población interesada en el tema son mujeres solteras con grado académico de licenciatura y que llevan relativamente poco tiempo de experiencia clínica $(6$ meses a 5 años) y con una edad promedio de 30.0 años, lo cual es la gente joven recién egresada quien se interesa por la atención del paciente terminal y en quien podríamos incidir para promover que los cuidados paliativos de difunda en la medicina y áreas afines a la salud, como la psicología, trabajo social, enfermería y odontología.

Los resultados de esta investigación cuya finalidad fue analizar la confiabilidad y el análisis de factor de un instrumento que nos ayudara a medir las actitudes ante los Cuidados Paliativos, nos Ileva a sugerir que la Escala de Actitudes ante los Cuidados Paliativos (EACP) es un instrumento capaz de identificar las actitudes ante esta rama de la Medicina que se encarga de la atención de los pacientes en fase avanzada

\section{Figura 1. Gráfica de componentes en espacio rotada de la Escala de Actitudes ante los Cuidados Paliativos (EACP)}

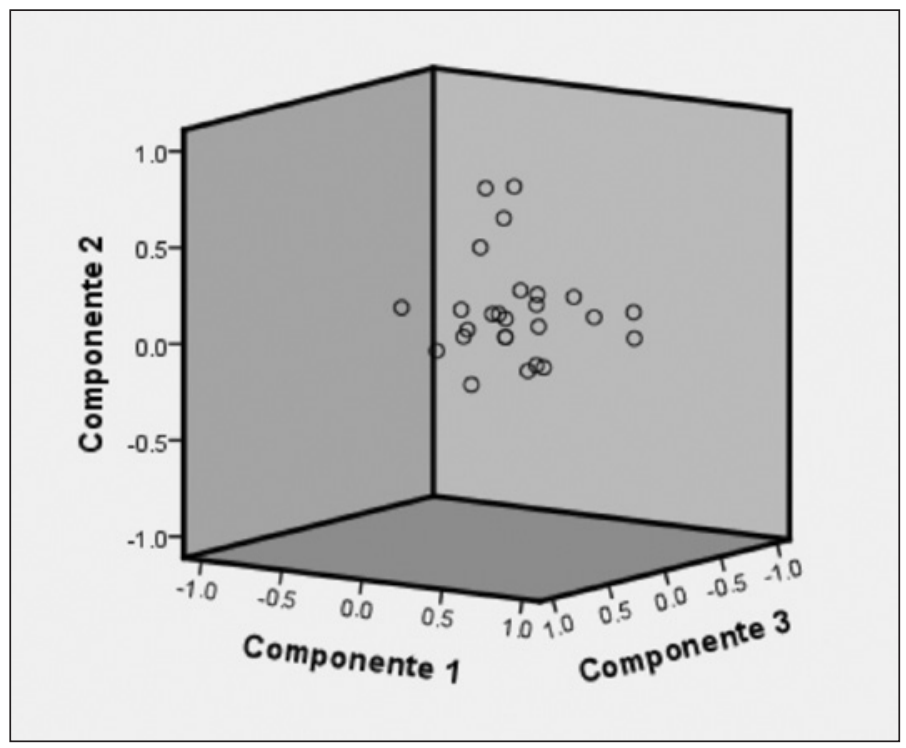


de las enfermedades cronico degenerativas y que requieren de una atención integral. De acuerdo a las normas de desarrollo de estudios instrumentales ${ }^{(30)}$, la escala cumple con las propiedades psicométricas que hacen de este instrumento una herramienta confiable para ser utilizado en el campo de la psicología, la medicina y otras áreas de la salud.

La escala tiene una buena fiabilidad, índices de validez y buen ajuste de los datos. La puntuación de Confiabilidad $(0,807)$ es similar a las obtenidas en los estudios realizados por Kain ${ }^{(20,21)}$ con un aplha de cronbach de 0,80 y Kumar, Anand y Siso$\mathrm{dia}^{(22)}$ con 0,90 .

Cabe señalar que el análisis factorial obtuvo 6 factores que engloban: el proceso de atención, las experiencias del personal de salud ante la muerte, la toma de decisiones, los aspectos sociales y la percepción profesional frente a la muerte. Sin embrago debe considerarse los reactivos 26 y 27 que deben ser replanteados de manera diferente para obtener un puntaje apha de manera positiva.

Por lo anterior podemos concluir que la Escala de Actitudes ante los Cuidados Paliativos (EACP) es un instrumento con condiciones psicométricas para ser considerado en futuras investigaciones.

\section{LIMITACIONES Y SUGERENCIAS}

Dentro de las limitaciones principales son que el test es aplicable principalmente a personal de salud que ya cuente en sus unidades de trabajo con un área de atención en cuidados paliativos y sesgo de la población lo cual limita la aplicación y uso en escenarios distintos.

También se sugiere con respecto al muestreo, considerar la posibilidad de realizar muestreos aleatorios o estratificados para que los datos puedan ser generalizables.

Además de considerar poder realizar el análisis factorial complementario, ya que nos permitiría ver el comportamiento de los 6 factores de forma gráfica y visual.

\section{AGRADECIMIENTOS:}

El presente proyecto fue aprobado por la Unidad de posgrado de la Universidad Chapultepec como parte de la Tesis Doctoral.

\section{REFERENCIAS BIBLIOGRÁFICAS}

1. Sabater, F. El valor de educar. Barcelona: Ed. Ariel, 1997.

2. Gordón M. Singer PA. Decisiones y cuidados al final de la vida. Lancet 1995; 27:411-4.

3. World Health Organization, (OMS). New guide on palliative care services for people living with advanced cancer. 2007. [Acceso 10 de octubre de 2015]. Disponible en: http://www.who.int/mediacentre/news/notes/2007/np31/en/

4. Billings A. What is palliative care? J Palliat Med 1998; 1:73- 81.

5. Emmanuel L. Palliative care I: providing care. Clin Geriatr Med 2004; 20:11-13.

6. Goldberg L. Psychologic issues in palliative care: Depression, anxiety, agitation, and delirium. Clin Fam Pract 2004; 6:441-70.

7. Weber M, Schmiedel S, Nauck F, AltEpping, B. Knowledge and attitude of final-year medical students in Germany towards palliative care-an interinstitutional questionnaire-based study. BMC PaIliat Care 2011; 10:19. Doi:10.1186/1472684X-10-19.

8. Bowden J, Dempsey K, Boyd K, Fallon M, Murray SA. Are newly qualified doctors prepared to provide supportive and endof-life care? A survey of Foundation Year 1 doctors and consultants. J R Coll Physicians Edinb 2013; 43:24-8. Doi: 10.4997/ JRCPE.2013.105.

9. Snyder S, Hazelett S, Allen K, Radwany S. Physician knowledge, attitude, and experience with advance care plan- 
ning, palliative care, and hospice results of a primary care survey. Am J Hosp Palliat Med 2013; 30:419-424. Doi: 10.1177/1049909112452467.

10. Chen E, McCann JJ, Lateef OB. Attitudes toward and experiences in end-oflife care education in the intensive care unit a survey of resident physicians. Am J Hosp Palliat Med 2014; 32:738-44. Doi: 10.1177/1049909114539038.

11. Kavalieratos D, Mitchell EM, Carey TS, Dev S, Biddle AK, Reeve BB, et al.. "Not the 'grim reaper service"': An assessment of provider knowledge, attitudes, and perceptions regarding palliative care referral barriers in heart failure. J Am Heart Assoc 2014; 3:1-11. Doi: 10.1161/ JAHA.113.000544.

12. Schaefer KG, Chittenden EH, Sullivan AM, Periyakoil VS, Morrison LJ, Carey EC, Sanchez-Reilly S, et al. Raising the bar for the care of seriously ill patients: Results of a national survey to define essential palliative care competencies for medical students and residents. Acad Med 2014; 89:1024-31. Doi:10.1097/ ACM.0000000000000271.

13. Twamley K, Kelly P, Moss R, Mancini A, Craig F, Koh, Polonsky $\mathrm{R}$ et al. Palliative care education in neonatal units: Impact on knowledge and attitudes. BMJ Support Palliat Care 2013; 3:213-20. Doi: 10.1136/ bmjspcare-2012-000336

14. Dietz I, Borasio GD, Med DP, Molnar C, Müller-Busch C, Plog A, Schneide C. et al. Errors in palliative care: Kinds, causes, and consequences: A pilot survey of experiences and attitudes of palliative care professionals. J Palliat Med 2013; 16:74-81. Doi: 10.1089/jpm.2012.0272

15. Loucka M, Pasman RH, Brearley SG, Payne SA, Onwuteaka-Philipsen B. Self-reported knowledge, attitudes, and behaviour towards hospice care and how are these related to training in palliative care: An online survey among oncologists in the Czech Republic and Slovakia. Progress in
Palliative Care 2013. Doi:10.1179/174329 1X13Y.0000000067.

16. Rivera MR, Torres FS. Physician attitudes on the provision of palliative care in puerto rican emergency departments. J Palliative Care Med 2015; 5:2-5. Doi:10.4172/21657386.10001201

17. Cherny N, Catane R. Attitudes of medical oncologists toward palliative care for patients with advanced and incurable cancer. Report on a survey by the European Society of Medical Oncology Taskforce on Palliative and Supportive Care. Cancer 2003; 98:2502-10. Doi:10.1002/cncr.11815

18. Cortezzo DE, Sanders MR, Brownell E, Moss K. Neonatologists' perspectives of palliative and end-of-life care in neonatal intensive care units. J Perinatol 2013; 33:731-5. Doi:10.1038/jp.2013.38

19. Frey R, Gott M, Banfield, R, Campbell $\mathrm{T}$. What questionnaires exist to measure the perceived competence of generalists in palliative care provision? A critical literature review. BMJ Support Palliat Care, 2011; 1:19-32. Doi:10.1136/bmjspcare-2011-000028

20. Kain V. Pilot Study of an Instrument to measure attitudes to palliative care practice in neonatal intensive care nursing. Collagian 2007; 14:16-20. Doi: 10.1016/ S1322-7696(08)60568-5

21. Kain V, Gardner G, Yates P. Neonatal paIliative care attitude scale: Development of an instrument to measure the barriers to and facilitators of palliative care in neonatal nursing. Pediatrics 2009; 123:e207-13. Doi: 10.1542/peds.2008-2774.

22. Kumar SP, Jim A, Sisodia V. Effects of palliative care training program on knowledge, attitudes, beliefs and experiences among student physiotherapists: A preliminary quasi-experimental study. Indian J Palliat Care 2011; 17:47-53. Doi: 10.4103/09731075.78449

23. Chen $\mathrm{CH}$, Huang $\mathrm{HL}$, Liu HY, Lee SY, Wu YC, Chang, YC, Peng NH. To explore the neonatal nurses' beliefs and attitu- 
des towards caring for dying neonates in Taiwan to explore the neonatal nurses' beliefs and attitudes towards caring for dying neonates in Taiwan. Matern Child Health J. 2013; 17:1793-801. Doi: 10.1007/ s10995-012-1199-0

24. Al-Kindi S, Zeinah A, Hassan A. Palliative care knowledge and attitudes among oncology nurses in Qatar. Am J Hosp Palliat Care 2014; 31:469-74. Doi: 10.1177/1049909113489874

25. Frommelt $\mathrm{KH}$. The effects on death education on nurses' attitudes toward caring for terminally ill persons and their families. Am J Hosp Palliat Care 1991; 8:37-43. Doi: 10.5430/jnep.v4n3p18

26. Frommelt $\mathrm{KH}$. Attitudes toward care of the terminally ill: An educational intervention. Am J Hosp Palliat Care 2003; 20:13-22. Doi:10.1177/104990910302000108

27. Leombruni P, Miniotti M, Bovero A, Zizzi F, Castelli L, Torta R. Attitudes toward caring for dying patients: An overview among Italian nursing students and preliminary psychometrics of the FATCOD-B scale. J Nurs Educ Pract 2014; 4:188-96. Doi:10.5430/jnep.v4n3p188

28. Mikulic MM. Construcción y Adaptación de pruebas psicológicas. Archivos de trabajos de la Universidad de Buenos Aires -Facultad de Psicología, 2012. [Acceso el 11 de mayo de 2012]. Disponible en: http://www.psi.uba.ar/academica/carrerasdegrado/psicologia/informacion_adicional/obligatorias/059_psicometricas1/tecnicas_psicometricas/archivos/f2.pdf

29. Aiken L. Test Psicológicos y Evaluación. México: Prentice Hall. 2003.

30. Carretero-Dios, H y Pérez, H. Normas para el desarrollo y revisión de estudios instrumentales: consideraciones sobre la selección de test en la investigación psicológica. International J Clin Health Psychol 2007; 7:863-82. 